\title{
Convergence of Vortex Methods for Euler's Equations
}

\author{
By Ole Hald and Vincenza Mauceri Del Prete
}

\begin{abstract}
A numerical method for approximating the flow of a two dimensional incompressible, inviscid fluid is examined. It is proved that for a short time interval Chorin's vortex method converges superlinearly toward the solution of Euler's equations, which govern the flow. The length of the time interval depends upon the smoothness of the flow and of the particular cutoff. The theory is supported by numerical experiments. These suggest that the vortex method may even be a second order method.
\end{abstract}

Introduction. In this paper we will prove the convergence of Chorin's vortex method for the flow of a two dimensional, inviscid fluid. The flow is governed by Euler's equations, which can be reduced to a scalar equation, the vorticity equation. In the classical point vortex method, studied by Rosenhead [10] and Westwater [13], it is assumed that the vorticity is concentrated at a number of points. This corresponds to approximating the vorticity by a sum of delta-functions. A point vortex is then moved by the velocity field induced by the other point vortices. However, the velocity field becomes unbounded near a point vortex, and this leads to a spurious interaction of neighboring vortices. This effect is not present in the original calculations by Rosenhead and Westwater, possibly because of the small number of vortices used or the limited accuracy of their calculations, see [3]. Recent experiments by Takami [12] and Moore [9], using a large number of vortices, indicate that the classical point vortex method is unreliable. To improve the vortex method Chorin [2] smoothes out the velocity field in a circle with center at the point vortex and radius $\delta$. This can be interpreted as approximating the vorticity by a sum of functions with small support, thus replacing the point vortices with blobs of vorticity.

There is considerable difference of opinion as to the optimal smoothing. Shestakov [11] follows Chorin [2] and takes $2 \pi \delta$ equal to the average distance $\beta$ between the vortices along the boundary on which the vortices are created. Depending upon the time step in the numerical solution of the associated ordinary differential equations, $\delta$ will be much larger than the average distance between the vortices in the direction normal to the boundary. On the other hand, Milinazzo and Saffman [8] believe that the cutoff $\delta$ should be as small as possible and take $\delta$ equal to $\beta / 50$. Finally, Chorin and Bernard [3] have observed that the results of the computations are quite insensitive to the exact details of the smoothing. Our analysis indicates that the optimal cutoff depends upon the smoothness of the flow under consideration, and that for smooth flows $\delta$ should be of order $\beta^{2 / 3}$. This implies that the cutoff $\delta$ tends 
to zero more slowly than the average distance $\beta$ between the vortices. With $\delta=\beta^{2 / 3}$ the rate of convergence is roughly speaking $\beta^{4 / 3}$, and this has been confirmed by numerical tests. However, our choice of $\delta$ is not optimal because numerical experiments with different kinds of smoothing show that the convergence of the vortex method can actually be of the second order.

The convergence of Chorin's method has already been considered by Dushane [4]. However, his proof is incorrect, and the obvious modifications do not eliminate the problem. Our proof follows the general outline of Dushane but introduces two new ideas. First, we do not assume that the cutoff $\delta$ and the average distance $\beta$ between the vortices are of the same order. Secondly, we do not compare the position of the point vortices to the streamlines of the flow, but rather to the center of mass (the centroid) of small blobs which move with the fluid. These changes lead to an improved estimate for the truncation error and this salvages the proof.

In one respect our result is less than satisfying. It can be shown that the solution of the Euler equations for a two dimensional flow exists for all time (see Wolibner [14], McGrath [7] and Kato [6]). However, we have only been able to prove the convergence of Chorin's method for a small time interval. The length of this interval depends on the Hölder continuity of the vorticity and on the details of the smoothing. Otherwise, our proof is quite economical. For example, we do not require more smoothness of the flow than that which is provided by the mathematical theory.

1. The Basic Equations. In this section we will present the vortex method and discuss different choices of smoothing.

The vorticity equations for a two dimensional incompressible, inviscid flow is

$$
\xi_{t}+(\mathbf{u} \cdot \nabla) \xi=0
$$

where $\mathbf{u}=(u, v)$ is the velocity field, $\xi=$ curl $\mathbf{u}$ is the vorticity, and $t$ is the time. Since the flow is incompressible, the divergence of $\mathbf{u}$ is equal to zero, and we may express $u$ and $v$ in terms of the stream function $\psi$ as follows

$$
\begin{gathered}
\Delta \psi=-\xi, \\
u=\psi_{y}, \quad v=-\psi_{x} .
\end{gathered}
$$

We will assume that $\xi$ has compact support and that $\mathbf{u}$ vanishes at infinity. The solution of Eq. (1.2) is then determined up to an additive constant and is given by the convolution $\psi=G * \xi$ where $G=-1 /(2 \pi) \log r$ with $r^{2}=x^{2}+y^{2}$.

It follows from Kelvin's theorem that the integral of the vorticity in a material blob is constant as the blob moves with the fluid (see [1, p. 274]). It is, therefore, natural to partition the support of $\xi$ into nonoverlapping blobs $B_{j}$ and to assign the vorticity in each blob to a single point $z_{j}$. This corresponds to approximating the vorticity by 


$$
\xi \sim \sum_{j} \kappa_{j} \delta\left(z-z_{j}\right)
$$

where $\kappa_{j}=\int_{B_{j}} \xi$ and $z=\left(\begin{array}{l}x \\ y\end{array}\right)$. The sum converges to $\xi$ in the sense of distributions as the diameter of the blobs tends to zero. To approximate the stream function, we smooth the kernel $G$ near the origin, thus obtaining $G_{\delta}$. The first two of the following examples have been used in practice

$$
\begin{aligned}
& G \sim G_{\delta} \equiv \frac{1}{2 \pi}\left[1-\frac{r}{\delta}-\log \delta\right], \\
& G \sim G_{\delta} \equiv \frac{1}{2 \pi}\left[\frac{1}{2}\left(1-\frac{r^{2}}{\delta^{2}}\right)-\log \delta\right], \\
& G \sim G_{\delta} \equiv \frac{1}{2 \pi}\left[\frac{3}{2}\left(1-\frac{r^{2}}{\delta^{2}}\right)-\frac{2}{3}\left(1-\frac{r^{3}}{\delta^{3}}\right)-\log \delta\right],
\end{aligned}
$$

for $r \leqslant \delta$, and $G_{\delta}=G$ for $r>\delta$ (see [2], [3] , [8] and [11]). It is straightforward to extend this list by requiring that the higher derivatives of $G_{\delta}$ are continuous at $r=\delta$. We will show that the vortex method converges provided $G_{\delta}$ is a smooth function of $r$ for $r<\delta$ and that the derivative of $G_{\delta}$ with respect to $r$ is Lipschitz continuous. By combining the approximation of $\xi$ with the smoothing of $G$, we obtain an approximation of the stream function, namely

$$
\psi \sim \sum G_{\delta}\left(z-z_{j}\right) \kappa_{j} .
$$

The distribution of vorticity at later times is obtained by letting the point vortices move with the fluid. Thus, by combining Eq. (1.3) with the approximation (1.7) we get

$$
\begin{gathered}
\dot{x_{i}}=\sum_{j \neq i} \partial_{y} G_{\delta}\left(z_{i}-z_{j}\right) \kappa_{j}, \\
\dot{y_{i}}=-\sum_{j \neq i} \partial_{x} G_{\delta}\left(z_{i}-z_{j}\right) \kappa_{j},
\end{gathered}
$$

where $z_{i}=\left(\begin{array}{l}x_{i} \\ y_{i}\end{array}\right)$ is the position of the $i$ th point vortex. Note that the sums are taken over $j$ different from $i$. In Chorin's method $G_{\delta}$ is given by (1.4) and the system of ordinary differential equations may have a unique solution only for a short time because two point vortices may collide. However, if $\partial_{r} G_{\delta}$ is Lipschitz continuous and vanishes at the origin as in (1.5) or (1.6), then the solution exists for all time.

The above presentation of the vortex method is mathematically oriented. However, the original derivation and justification of the vortex method is based on physical arguments (see [2]). Let $\zeta_{\delta}$ be defined by $\Delta G_{\delta}=-\zeta_{\delta}$, where the derivatives are taken in the sense of distributions. Then $\zeta_{\delta}$ has compact support and will approximate the delta function. For (1.4) to (1.6) we see that $\zeta_{\delta}$ is equal to $1 /(2 \pi r \delta), 1 /\left(\pi \delta^{2}\right)$ and $3(1-r / \delta) /\left(\pi \delta^{2}\right)$ for $r<\delta$ and zero otherwise. Thus, $G_{\delta}$ can be interpreted as the stream function corresponding to a small circular blob with the vorticity $\zeta_{\delta}$ and (1.7) is, therefore, the stream function corresponding to the vorticity distribution 


$$
\xi_{\delta}=\sum_{j} \zeta_{\delta}\left(z-z_{j}\right) \kappa_{j}
$$

This interpretation is due to Chorin [2]. It can be shown that if $G_{\delta}$ is given by (1.6), then $\xi_{\delta}$ is continuous and converges uniformly to $\xi$, provided the diameter of the blobs $B_{j}$ tends to zero faster than $\delta$. This implies an ever increasing overlap of the circular blobs.

2. Properties of the Flow. In this section we will show that the distance between two points of the flow can be bounded from above and below in terms of the initial distance of the points. The flow is then partitioned in nonoverlapping blobs which move with the fluid. We will estimate the distance between the centroid (the center of mass) of a blob and the path of the material element which coincides with the centroid initially. Finally, we will prove that the centroids do not collide for a finite time, provided the partition is sufficiently fine.

Throughout this paper we will assume that the vorticity $\xi$ is differentiable with respect to $t$ and that $\xi$ and the partial derivatives of $\mathbf{u}$ are uniformly Hölder continuous with exponent $\alpha$, i.e. $\xi$ and the components of $\nabla \mathbf{u}$ satisfy

$$
\left|\xi\left(z_{1}\right)-\xi\left(z_{2}\right)\right| \leqslant H\left|z_{1}-z_{2}\right|^{\alpha}
$$

for all $z_{1}$ and $z_{2}$ in $R^{2}$. Here $H$ and $\alpha$ do not depend on $t$ for $0 \leqslant t \leqslant T$. For the related problem of a two dimensional flow in a bounded, possibly multi-connected domain with smooth boundary, Kato has shown that our assumptions are satisfied [6]. We begin with an estimate of the expansion and the contraction of the flow.

LEMMA 1 (DuShANe). Let $z_{1}(t)$ and $z_{2}(t)$ be the path of two material points of the flow. Then for $0 \leqslant t \leqslant T$,

$$
C_{1}^{-1}\left|z_{1}(0)-z_{2}(0)\right| \leqslant\left|z_{1}(t)-z_{2}(t)\right| \leqslant C_{1}\left|z_{1}(0)-z_{2}(0)\right|,
$$

where $|z|=\sqrt{x^{2}+y^{2}}$. The constant $C_{1}$ is independent of $z_{1}$ and $z_{2}$ but depends on $T$ and the flow under consideration.

Proof. Let $z=\left(\begin{array}{l}x \\ y\end{array}\right)$ be a point in $R^{2}$. The path of a material element is obtained by solving the ordinary differential equation $\dot{z}=\mathrm{u}(z, t)$, with initial conditions $z(0)=$ $z_{0}$. By using the fundamental theorem of calculus we see that

$$
\dot{z}_{1}-\dot{z}_{2}=\mathrm{u}\left(z_{1}\right)-\mathrm{u}\left(z_{2}\right)=A\left(z_{1}-z_{2}\right)
$$

where $A=\int_{0}^{1} \nabla \mathbf{u}\left(z_{2}+\theta\left(z_{1}-z_{2}\right)\right) d \theta$. Since $\xi$ is Hölder continuous and has compact support, we can estimate the. 2 -norm of $A$ by $m=\max |\nabla \mathbf{u}|$. The maximum is taken over $z$ in $R^{2}$ and $t$ in $[0, T]$. Let $F=\left|z_{1}-z_{2}\right|^{2}$. It follows from Eq. (2.2) that $|\dot{F}| \leqslant 2 m F$. By integrating this differential inequality we obtain

$$
e^{-2 m t} F(0) \leqslant F(t) \leqslant e^{2 m t} F(0) \text {. }
$$

The proof is completed by taking $C_{1}=e^{m T}$.

We consider now the support of the vorticity at time $t=0$. We partition the support in a finite number of nonoverlapping squares $B_{i}$ and let $\beta$ be the length of the 
sides. For $t>0$ the squares move with the fluid and change their shape, but since the flow is incompressible the area of the blobs $B_{j}(t)$ remains constant. By using Lemma 1 we make the following

Observation. Let $C_{2}=\sqrt{2} C_{1}$. The diameter of $B_{j}(t)$ is less than $C_{2} \beta$ for $0 \leqslant$ $t \leqslant T$.

A rough description of the position of the blob $B_{j}$ at time $t$ is provided by its center of mass, the centroid. It is defined by

$$
z_{j}=\beta^{-2} \int_{B_{j}(t)} z d x d y,
$$

where $z=\left(\begin{array}{l}x \\ y\end{array}\right)$. Note that $\beta^{2}$ is the area of $B_{j}(t)$. Observe also that $z_{j}$ depends upon $t$ because the centroid follows the blob as the blob moves with the fluid. To find $\dot{z}_{j}$ we introduce the mapping $\Phi_{t}$. Here $\Phi_{t}$ maps the position of a material point at time $t=0$ to the position of the point at time $t$, i.e. $\Phi_{t}: z(0) \rightarrow z(t)$. Since the path of a material element is given by $\dot{z}=\mathbf{u}(z)$, it follows from Lemma 1 and the incompressibility of the flow that $\Phi_{t}$ is a one-to-one, measure preserving transformation of $R^{2}$ onto itself, and its Jacobian is equal to one. We can now use the change of variables formula to compute $\dot{z}_{j}$ and get

$$
\dot{z}_{j}=\beta^{-2} \int_{B_{j}(t)} \mathrm{u}(z) d x d y .
$$

Thus, the centroid moves with the average velocity of the blob. It is, therefore, natural to compare the path of the centroid to the path of the material element which coincides with the centroid initially.

LEMMA 2. Let $z_{j}(t)$ be the path of the centroid of $B_{j}(t)$ and let $z(t)$ be the path of the material element for which $z(0)=z_{j}(0)$. Then

$$
\left|z_{j}(t)-z(t)\right| \leqslant C_{4} \beta^{1+\alpha}
$$

for $0 \leqslant t \leqslant T$. The constant $C_{4}$ is independent of $B_{j}$ and $\beta$ but depends on $T$ and the flow.

Remark. The centroid is always in the convex hull of $B_{j}(t)$, but by combining Lemmas 1 and 2 , we see that it is actually an interior point of the blob for $\beta$ sufficiently small.

Proof. Let $B_{j}=B_{j}(t)$. It follows from Taylor's formula with remainder that

$$
\dot{z}_{j}=\beta^{-2} \int_{B_{j}}\left\{\mathbf{u}\left(z_{j}\right)+\int_{0}^{1}\left[\nabla \mathbf{u}\left(z_{j}+\theta\left(z^{\prime}-z_{j}\right)\right)-\nabla \mathbf{u}\left(z_{j}\right)\right] d \theta \cdot\left(z^{\prime}-z_{j}\right)\right\} d z^{\prime},
$$

where $d z^{\prime}=d x^{\prime} d y^{\prime}$ and we have used that $\int_{B_{j}} z^{\prime}-z_{j}$ vanishes. It is this property which makes the centroid such a powerful tool. To estimate the last term in the above equation, we remember that $\nabla \mathbf{u}$ is Hölder continuous. Since $\left|z^{\prime}-z_{j}\right|$ is less than the diameter of $B_{j}$ for all $z^{\prime}$ in $B_{j}$, we find by using Observation 1 that

$$
\left|\dot{z}_{j}-\mathbf{u}\left(z_{j}\right)\right| \leqslant 2 H\left(\operatorname{diam} B_{j}\right)^{1+\alpha} \leqslant C_{3} \beta^{1+\alpha},
$$


where $C_{3}=2 H C_{2}^{1+\alpha}$. We consider now the difference $z_{j}-z$. Since the path of the material element satisfies $\dot{z}=\mathbf{u}(z)$, we conclude by using the inequality (2.3) and the fundamental theorem of calculus that

$$
\begin{aligned}
\dot{z}_{j}-\dot{z} & =\mathbf{u}\left(z_{j}\right)-\mu(z)+C_{3} \beta^{1+\alpha} \mathbf{e} \\
& =A\left(z_{j}-z\right)+C_{3} \beta^{1+\alpha} \mathbf{e},
\end{aligned}
$$

where $\mathrm{e}$ is a vector with norm less than one. Let $F=\left|z_{j}-z\right|$. This function is differentiable from the right (see Hartmann [5, p. 26]). Since the 2-norm of $A$ is less than $m=\max |\nabla \mathbf{u}|$, we obtain the estimate $\dot{F} \leqslant m F+C_{3} \beta^{1+\alpha}$. By integrating this differential inequality we find

$$
F \leqslant C_{3} \beta^{1+\alpha} \frac{e^{m T}-1}{m}=C_{4} \beta^{1+\alpha}
$$

This completes the proof.

In the convergence proof for the vortex method, we estimate the distance between the point vortices and the centroids of the blobs. Thus we presume the existence of a solution of Eqs. (1.8) and (1.9). To prove that the point vortices do not collide for a finite time which is independent of $\beta$ we need

Corollary 1. Let $z_{i}(t)$ and $z_{j}(t)$ be the centroids of $B_{i}(t)$ and $B_{j}(t)$. If $\left|z_{i}(0)-z_{j}(0)\right| \geqslant \beta$, then

$$
\left|z_{i}(t)-z_{j}(t)\right| \geqslant c_{2} \beta
$$

for $0 \leqslant t \leqslant T$, provided $\beta$ is sufficiently small. The constant $c_{2}$ is positive and depends on $T$ and the flow.

Proof. Let $z^{(i)}$ and $z^{(j)}$ be the paths of the material elements which coincide with $z_{i}$ and $z_{j}$ initially. By using the triangle inequality and Lemmas 1 and 2 , we get

$$
\begin{aligned}
\left|z_{i}-z_{j}\right| & \geqslant\left|z^{(i)}-z^{(j)}\right|-\left|z_{i}-z^{(i)}\right|-\left|z_{j}-z^{(j)}\right| \\
& \geqslant\left(C_{1}^{-1}-2 C_{4} \beta^{\alpha}\right) \beta .
\end{aligned}
$$

To complete the proof we let $\beta$ be less than $\left(4 C_{1} C_{4}\right)^{-\alpha}$ and take $c_{2}=\left(2 C_{1}\right)^{-1}$.

3. Consistency and Stability. For linear differential equations it is well known that consistency plus stability implies convergence for all time. For the vortex method, we prove that consistency plus weak instability implies convergence for a short time. In this section we will first consider the truncation error and then the stability of the vortex method.

Let $\mathbf{u}$ be the velocity field at time $t$. It follows from Eq. (1.3) that $\mathbf{u}=\mathbf{K} * \xi$ where $\mathbf{K}=\left(\begin{array}{c}\partial y \\ -\partial x\end{array}\right) G$. Similarly, we define $\mathbf{K}_{\delta}$ as $\left(\begin{array}{c}\partial y \\ -\partial x\end{array}\right) G_{\delta}$ and set $\mathbf{K}_{\delta}(0)=0$. We will assume that $\mathbf{K}_{\delta}$ is continuous for $z$ different from zero and that there exists a constant $C_{0}$ such that

$$
\left|\partial_{x}^{p} \partial_{y}^{q} \mathbf{K}_{\delta}(z)\right| \leqslant \begin{cases}C_{0} / \delta|z|^{p+q} & \text { for } 0<|z| \leqslant \delta, \\ C_{0} /|z|^{1+p+q} & \text { for }|z|>\delta,\end{cases}
$$


for all $p+q \leqslant 2$. For the cutoffs presented in (1.4) to (1.6), the constant $C_{0}$ is equal to $1 / \pi, 1 / \pi$ and $2 / \pi$, respectively. Note that $C_{0}$ cannot be less than $1 / \pi$ since $K_{\delta}=K$ for $|z|>\delta$. To estimate the truncation error for the vortex method, we need the following result:

LemMA 3. Let $z_{j}$ be the centroids of the blobs $B_{j}$ at time $t$ and let $D$ be the maximum of the diameter of the support of $\xi$ for $0 \leqslant t \leqslant T$. If $\xi$ and $\mathbf{K}_{\delta}$ satisfy the inequalities (2.1) and (3.1), then there exists a constant $C_{5}$ such that

$$
\left|\mathbf{u}(z)-\sum_{j} \mathbf{K}_{\delta}\left(z-z_{j}\right) \kappa_{j}\right| \leqslant C_{5}|\log \beta| \beta^{(1+\alpha) /(1+\alpha / 2)}
$$

for $0 \leqslant t \leqslant T$ and $\beta$ sufficiently small. The distance from $z$ to supp $\xi$ should be less than $D$, and $\delta$ equal to $\beta^{1 /(1+\alpha / 2)}$.

Remark. The factor $-\log \beta$ can be omitted for smooth cutoffs such as (1.5) and (1.6). By combining Lemma 3 with the inequality (2.3), we see that our estimate of the truncation error $\tau$ depends on the smoothness of the flow. If the vorticity $\xi$ is differentiable, then $\tau \sim \beta^{4 / 3}|\log \beta|$. This is an improvement of the estimate due to Dushane. In his paper [4] Dushane takes $\delta=O(\beta)$ and obtains $\tau \sim \beta|\log \beta|$. Also, the optimal cutoff depends upon the vorticity. If $\xi$ is smooth, then $\delta=\beta^{2 / 3}$. For highly irregular flows $\delta$ will be close to $\beta$. We choose $\delta=\beta^{1 /(1+\alpha / 2)}$, but since the theory below is an asymptotic theory, we could also have used $\delta=100 \beta^{1 /(1+\alpha / 2)}$. This would only change the constant $C_{5}$. Actually the vortex method will converge faster than linearly whenever $\delta=\beta^{p}$, where $1 /(1+\alpha)<p<1$.

Proof. Since the $B_{j}$ 's cover the supp $\xi$, we find

$$
\begin{aligned}
\mathbf{u}(z)= & \sum_{j} \mathbf{K}_{\delta}\left(z-z_{j}\right) \int_{B_{j}} \xi\left(z^{\prime}\right) d z^{\prime}+\sum_{j} \int_{B_{j}}\left[\mathbf{K}_{\delta}\left(z-z^{\prime}\right)-\mathbf{K}_{\delta}\left(z-z_{j}\right)\right] \xi\left(z^{\prime}\right) d z^{\prime} \\
& +\int_{\left|z-z^{\prime}\right|<\delta}\left[\mathbf{K}\left(z-z^{\prime}\right)-\mathbf{K}_{\delta}\left(z-z^{\prime}\right)\right]\left[\xi\left(z^{\prime}\right)-\xi(z)\right] d z^{\prime}
\end{aligned}
$$

where $d z^{\prime}=d x^{\prime} d y^{\prime}$. Note that the integral of $\mathbf{K}-\mathbf{K}_{\delta}$ over $\left|z-z^{\prime}\right|<\delta$ is zero. Since $\xi$ is Hölder continuous, we can estimate the last tern of Eq. (3.2) by $H\left(1+\pi C_{0}\right) \delta^{1+\alpha}$. To estimate the second term, we cover the supp $\xi$ by $N$ annuli, with center at $z$ and radii $r_{k-1}$ and $r_{k}$ where $r_{k}=k C_{2} \beta$ and $k=1,2, \ldots, N$. Here $N=1+\left[2 D / r_{1}\right]$, where $[a]$ is the greatest integer less than or equal to $a$. Let $I_{k}$ be those centroids $z_{j}$ for which $r_{k-1} \leqslant\left|z-z_{j}\right|<r_{k}$. Then the second term of Eq. (3.2) is

$$
S=\sum_{k=1}^{N} \sum_{z_{j} \in I_{k}} \int_{B_{j}}\left[\mathbf{K}_{\delta}\left(z-z^{\prime}\right)-\mathbf{K}_{\delta}\left(z-z_{j}\right)\right] \xi\left(z^{\prime}\right) d z^{\prime} .
$$

Note that $S$ is a vector with two components. Since $\mathbf{K}_{\delta}$ may not be continuous at the origin and the derivatives of $\mathbf{K}_{\delta}$ will be discontinuous at the circle of radius $\delta$, we divide the disk $\left|z-z^{\prime}\right|<r_{N}$ in four nonoverlapping regions. Let $n=1+\left[\delta / r_{1}\right]$. Region I consists of the first three annuli, and region II consists of the union of the fourth to the $n-2$ th annulus. Region III is the ring with radii $r_{n-2}$ and $r_{n+1}$, and region IV is the union of the remaining annuli up to the $N$ th. Similarly, we split the sum $S$ into four parts, $S_{\mathrm{I}}$ to $S_{\mathrm{IV}}$. 
We observe now that a blob cannot intersect more than two annuli at any time because its diameter is less than the distance between the circles. Since the area of each blob is constant, we conclude that the total area of the blobs with centroids in a given annulus cannot be larger than three times the area of that annulus.

To estimate $S_{\mathrm{I}}$ we choose $\beta$ such that $r_{4}<\delta$. This implies that $n \geqslant 5$. Since the blobs with centroids in region I cannot cover more than the first four annuli, we conclude by using the estimate (3.1) that

$$
\left|S_{\mathrm{I}}\right| \leqslant 32 \pi C_{0} C_{2}^{2} M \frac{\beta^{2}}{\delta},
$$

where $M$ is larger than $\max |\xi|$ for $0 \leqslant t \leqslant T$. In region II, $\mathbf{K}_{\delta}$ is a smooth function. Thus it follows from Taylor's formula with remainder that

$$
\begin{aligned}
S_{\mathrm{II}}=\sum_{k=4}^{n-2} \sum_{z_{j} \in I_{k}}[ & \nabla \mathbf{K}_{\delta}\left(z-z_{j}\right) \int_{B_{j}}\left(z^{\prime}-z_{j}\right)\left\{\xi\left(z^{\prime}\right)-\xi\left(z_{j}\right)\right\} d z^{\prime} \\
& +\int_{B_{j}} \sum_{p+q=2} \int_{0}^{1}(1-\theta) \partial_{x}^{p} \partial_{y}^{q} \mathbf{K}_{\delta}\left(z-z_{j}+\theta\left(z^{\prime}-z_{j}\right)\right) d \theta \\
& \left.\times\left(x^{\prime}-x_{j}\right)^{p}\left(y^{\prime}-y_{j}\right)^{q} \xi\left(z^{\prime}\right) d z^{\prime}\right],
\end{aligned}
$$

where we have used that $\int_{B} z^{\prime}-z_{j}=0$. According to the Observation following Lemma 1 , the diameter of $B_{j}$ is less than or equal to $r_{1}$. Since $\xi$ is Hölder continuous and the 2-norm of $\nabla \mathbf{K}_{\delta}$ is less than $\sqrt{2} C_{0} / \delta$, we see that

$$
\begin{aligned}
\left|S_{\mathrm{II}}\right| \leqslant & \sum_{k=4}^{n-2} \frac{\sqrt{2} C_{0}}{\delta(k-1) r_{1}} r_{1} \cdot H r_{1}^{\alpha} \cdot 3 \pi(2 k-1) r_{1}^{2} \\
& +\sum_{k=4}^{n-2} \frac{C_{0}}{\delta(k-2)^{2} r_{1}^{2}} \cdot \frac{1}{2} \cdot 2 r_{1}^{2} \cdot M \cdot 3(2 k-1) r_{1}^{2}
\end{aligned}
$$

To estimate the sums we use $n \leqslant 1+\delta / r_{1}$ and get after a straightforward, but lengthy calculation,

$$
\left|S_{\mathrm{II}}\right| \leqslant 10 \pi C_{0} C_{2}^{1+\alpha} H \beta^{1+\alpha}+6 \pi C_{0} C_{2}^{2} M \frac{\beta^{2}}{\delta} \cdot \log \left(\frac{2 \delta}{\beta}\right) .
$$

In region III we observe that $\mathbf{K}_{\delta}$ may not be differentiable, but it follows from the bound (3.1) that $\mathbf{K}_{\delta}$ is Lipschitz continuous with Lipschitz constant $\sqrt{2} C_{0} /\left(\delta r_{n-3}\right)$. We can, therefore, estimate $S_{\text {III }}$ by

$$
\begin{aligned}
\left|S_{\mathrm{III}}\right| & \leqslant \sum_{k=n-1}^{n+1} \sum_{z_{j} \in I_{k}} \frac{\sqrt{2} C_{0}}{\delta(n-3) r_{1}} \cdot r_{1} \cdot M \cdot\left|B_{j}\right| \\
& \leqslant 50 \pi C_{0} C_{2}^{2} M \frac{\beta^{2}}{\delta},
\end{aligned}
$$

where $\left|B_{j}\right|$ is the area of $B_{j}$. To estimate $S_{\text {IV }}$ we use the same approach as for $S_{\text {II }}$ and obtain 


$$
\left|S_{\mathrm{IV}}\right| \leqslant 9 \pi C_{0} C_{2}^{1+\alpha} H \beta^{1+\alpha} \log \left(\frac{3 D}{\delta}\right)+9 \pi C_{0} C_{2}^{2} \cdot M \frac{\beta^{2}}{\delta}
$$

We have now shown that the last two terms of (3.2) can be estimated in terms of the maximum of $\delta^{1+\alpha}, \beta^{2} / \delta$ and $\beta^{1+\alpha}$. Since $\beta<\delta$ we find the optimal choice of $\delta$ by letting $\delta^{1+\alpha} \sim \beta^{2} / \delta$ and this leads to $\delta=\beta^{1 /(1+\alpha / 2)}$. To complete the proof we add the estimates for $S_{\mathrm{I}}$ to $S_{\mathrm{IV}}$ to $H\left(1+\pi C_{0}\right) \delta^{1+\alpha}$, use that $\log (2 \delta / \beta)<$ $2 / 3|\log \beta|$ and $\log (3 D / \delta)<2|\log \beta|$ for $\beta$ sufficiently small and let

$$
C_{5}=20 \pi C_{0} C_{2}^{2}(H+5 M) \text {. }
$$

Lemma 4. Let $z_{j}$ be the centroids of the blobs $B_{j}$ at time $t$ and let $c_{2}$ be the constant in Corollary 1. If $\max _{j}\left|\widetilde{z}_{j}-z_{j}\right| \leqslant c_{2} \beta / 4$, then

$$
\max _{i} \sum_{j \neq i}\left|\mathbf{K}_{\delta}\left(\widetilde{z}_{i}-\widetilde{z}_{j}\right)-\mathbf{K}_{\delta}\left(z_{i}-z_{j}\right)\right|\left|\kappa_{j}\right| \leqslant C|\log \beta| \max _{j} \widetilde{z_{j}}-z_{j} \mid
$$

for $0 \leqslant t \leqslant T$ and $\beta$ sufficiently small. The constant $C$ depends on $T$ and the flow under consideration.

Remark. This stability result is a discrete analogue of a basic lemma in the mathematical theory for Euler's equations (see Wolibner [14], McGrath [7] and Kato [6]). The factor $|\log \beta|$ occurs also in the mathematical theory where it is replaced by $1-\log \left(\max _{j}\left|\widetilde{z}_{j}-z_{j}\right|\right)$. If this factor were not present, we could easily prove the convergence of the vortex method for all time. As it is, we get convergence for a short time only.

Proof. We will use the ideas and the notations from the preceding proof with minor modifications. Let $z_{i}$ be fixed and let $I_{k}$ be the set of $z_{j}$ in the $k$ th annulus. Since $\kappa_{j}=\int_{B_{j}} \xi$ we conclude that $\left|\kappa_{j}\right| \leqslant M\left|B_{j}\right|$, where $M \geqslant|\xi|_{\infty}$ for all $0 \leqslant t \leqslant T$ and $\left|B_{j}\right|$ is the area of $B_{j}$. It is, therefore, sufficient to estimate

$$
S=\sum_{k=1}^{N} \sum_{z} \in I_{k}\left|\mathbf{K}_{\delta}\left(\tilde{z_{i}}-\tilde{z}_{j}\right)-\mathbf{K}_{\delta}\left(z_{i}-z_{j}\right)\right|\left|B_{j}\right| .
$$

We partition the sum $S$ into four sums, $S_{\mathrm{I}}$ to $S_{\mathrm{IV}}$ according to regions I to IV. Let $E=\max _{j}\left|\widetilde{z}_{j}-z_{j}\right|$. In the lemma we have assumed that $E \leqslant c_{2} \beta / 4$. Since $c_{2}<1 / 2$, this certainly implies that $2 E<r_{1}$ where $r_{1}=C_{2} \beta$. Thus, by using the triangle inequality and Corollary 1 we find that

$$
c_{2} \beta / 2 \leqslant\left|z_{i}-z_{j}+\theta\left[\tilde{z}_{i}-z_{i}-\left(\tilde{z}_{j}-z_{j}\right)\right]\right| \leqslant\left|z_{i}-z_{j}\right|+r_{1}
$$

for $i \neq j$ and $0 \leqslant \theta \leqslant 1$. This shows that all the points on the line segment between $\widetilde{z}_{i}-\widetilde{z}_{j}$ and $z_{i}-z_{j}$ are different from zero and lie in the first four annuli for $z_{j}$ in region I. To estimate $S_{\mathrm{I}}$ we let $w_{j}=\tilde{z}_{i}-z_{i}-\left(\tilde{z}_{j}-z_{j}\right)$ and by using the fundamental theorem of calculus we get

$$
\left|S_{\mathrm{I}}\right| \leqslant \sum_{k=1}^{3} \sum_{z} \in I_{k} \int_{0}^{1}\left|\nabla \mathbf{K}_{\delta}\left(z_{i}-z_{j}+\theta w_{j}\right)\right| d \theta\left|w_{j}\right|\left|B_{j}\right| .
$$

By combining the estimates (3.1) and (3.4), we see that 


$$
\left|S_{\mathrm{I}}\right| \leqslant \sum_{z_{j} \in I_{1} \cup I_{2} \cup I_{3}} \frac{\sqrt{2} C_{0}}{\delta c_{2} \beta / 2} 2 E\left|B_{j}\right| \leqslant 128 \pi C_{0} C_{2}^{3} \frac{\beta}{\delta} E,
$$

where we have used $c_{2}^{-1}=\sqrt{2} C_{2}$. The estimates of $S_{\mathrm{II}}$ to $S_{\mathrm{IV}}$ are derived in much the same manner. If $z_{j}$ is in $I_{k}$ with $k>3$, then the lower bound in (3.4) can be replaced by $r_{k-2}$ and the upper bound can be replaced by $r_{k+1}$. Since $n \leqslant 1+$ $\delta / r_{1}$, we conclude that

$$
\left|S_{\mathrm{II}}\right| \leqslant \sum_{k=4}^{n-2} \frac{\sqrt{2} C_{0}}{\delta(k-2) r_{1}} 2 E 3 \pi(2 k-1) r_{1}^{2} \leqslant 30 \pi C_{0} E
$$

If $z_{j}$ is in region III, then $\nabla \mathbf{K}_{\delta}$ may have a discontinuity on the line connecting $\widetilde{z}_{i}-\widetilde{z}_{j}$ and $z_{i}-z_{j}$. However, since all points on this line segment lie outside the disk with center at $z_{i}$ and radius $r_{n-3}$, we see that $\mathbf{K}_{\delta}$ is Lipschitz continuous with Lipschitz constant $\sqrt{2} C_{0} /\left(\delta r_{n-3}\right)$. Thus,

$$
\left|S_{\mathrm{III}}\right| \leqslant 64 \pi C_{0} C_{2} \frac{\beta}{\delta} E
$$

Finally, by using the fundamental theorem of calculus once more, we can estimate

$$
\left|S_{\mathrm{IV}}\right| \leqslant \sum_{k=n+2}^{N} \frac{\sqrt{2} C_{0}}{(k-2)^{2} r_{1}^{2}} 2 E \cdot 3 \pi(2 k-1) r_{1}^{2} \leqslant 24 \pi C_{0} \log \left(\frac{3 D}{\delta}\right) E .
$$

To complete the proof we sum the estimates for $S_{\mathrm{I}}$ to $S_{\mathrm{IV}}$, multiply the result by $M$ and use that $\beta / \delta$ is less than one. Since $C_{2}>\sqrt{2}$ and $\log (3 D / \delta)<2|\log \beta|$ for $\beta$ sufficiently small, we may choose the constant $C$ as

$$
C=190 \pi C_{0} C_{2}^{3} M
$$

4. Convergence Results. In this section we will establish the convergence of the vortex method on three different levels. The basic result is that the path of the point vortices computed by (1.8) and (1.9) converges toward the path of the centroid of the material elements. This implies the convergence of the induced velocity field and also the convergence of the vorticity distribution.

THEOREM 1. Let $z_{j}$ be the centroid of $B_{j}$ and let $\tilde{z}_{j}$ be the solution of Eqs. (1.8), (1.9). There exist two constants, $C_{6}$ and $T_{0}$, such that

$$
\max _{j}\left|\tilde{z}_{j}-z_{j}\right| \leqslant C_{6} \beta^{(1+\alpha) /(1+\alpha / 2)-C t}
$$

for $0 \leqslant t \leqslant T_{0}$ and $\beta$ sufficiently small. The constant $C$ is given by Eq. (3.5).

Remark. It follows from Lemma 2 that the centroids $z_{j}$ in the theorem can be replaced by the paths of the material elements with the same initial positions as the $z_{j}$. Thus the paths of the point vortices will approximate the streamlines if the flow is stationary.

Proof. By combining Eqs. (1.8), (1.9) with Lemma 3 and the inequality (2.3), we see that 


$$
\left(\widetilde{z_{i}}-z_{i}\right)=\sum_{j \neq i}\left[\mathbf{K}_{\delta}\left(\widetilde{z}_{i}-\widetilde{z}_{j}\right)-\mathbf{K}_{\delta}\left(z_{i}-z_{j}\right)\right] \kappa_{j}-\theta\left[C_{3} \beta^{1+\alpha}+C_{5}|\log \beta| \beta^{\gamma}\right],
$$

where $|\theta| \leqslant 1$ and $\gamma=(1+\alpha) /(1+\alpha / 2)$. Let $E=\max _{j}\left|\widetilde{z}_{j}-z_{j}\right|$. Since $\widetilde{z}_{j}-z_{j}$ is differentiable with respect to $t$, it can be shown that $E(t)$ has a right derivative, which we denote by $\dot{E}$ (see $\left[5\right.$, p. 26]). Assume now that $E \leqslant c_{2} \beta / 4$. We can then apply Lemma 4 and get

$$
\dot{E} \leqslant C|\log \beta| E+\left(C_{3}+C_{5}\right)|\log \beta| \beta^{\gamma} .
$$

By integrating this differential inequality and using the estimates for $C, C_{0}, C_{3}$, and $C_{5}$, we obtain after some simplification

$$
\begin{gathered}
E(t) \leqslant\left(C_{3}+C_{5}\right)|\log \beta| \beta^{\gamma} \frac{e^{C|\log \beta| t}-1}{C|\log \beta|} \\
\leqslant \frac{1}{8} \frac{1}{C_{2}}\left(5+\frac{H}{M}\right) \beta^{\gamma-C t} .
\end{gathered}
$$

This estimate is only valid as long as $E \leqslant c_{2} \beta / 4$. In the proof of Corollary 1 , we took $c_{2}=\left(\sqrt{2} C_{2}\right)^{-1}$. Our requirement can, therefore, be rephrased as

$$
\beta^{\gamma-1-C t} \leqslant \frac{8}{4 \sqrt{2}}\left(5+\frac{H}{M}\right)^{-1}
$$

This inequality will certainly be satisfied for all $t$ in the interval $\left[0, T_{0}\right]$ provided that $T_{0}$ is strictly less than $T$ and $\alpha(2+\alpha)^{-1} C^{-1}$ and that $\beta$ is sufficiently small. The proof is now completed by taking $C_{6}=(5+H / M) /\left(8 C_{2}\right)$.

Let $\widetilde{z}_{j}$ be the position of the point vortices computed by $(1.8),(1.9)$. To approximate the velocity field $\mathbf{u}$ for the flow we will use

$$
\widetilde{\mathbf{u}}(z)=\sum_{j} \mathbf{K}_{\delta}\left(z-\widetilde{z}_{j}\right) \kappa_{j}
$$

This choice is natural, but it should be observed that $\tilde{\mathbf{u}}$ may not be continuous as $\mathbf{K}_{\delta}$ may have a discontinuity at the origin. Nevertheless, we will now show that $\tilde{\mathbf{u}}$ converges uniformly to $\mathbf{u}$ as $\beta$ tends to zero.

THEOREM 2. There exists a constant $C_{7}$ such that

$$
\max _{z \in R^{2}} \tilde{\mathbf{u}}(z)-\mathbf{u}(z)\left|\leqslant C_{7}\right| \log \beta \mid \beta^{(1+\alpha) /(1+\alpha / 2)-C t}
$$

for $0 \leqslant t \leqslant T_{0}$ if $\beta$ is sufficiently small and $\delta=\beta^{1 /(1+\alpha / 2)}$.

Remark. Dushane [4] obtains the estimate $|u-\widetilde{u}| \leqslant$ const $\beta^{2-C-\epsilon}$, which is better than ours, but his proof is incorrect.

Proof. Let the distance from $z$ to supp $\xi$ be less than $D$. It follows from Eq. (4.1) and Lemma 3 that

$$
\widetilde{\mathbf{u}}-\mathbf{u}=\sum_{j}\left[\mathbf{K}_{\delta}\left(z-\widetilde{z}_{j}\right)-\mathbf{K}_{\delta}\left(z-z_{j}\right)\right] \kappa_{j}-\theta C_{\mathbf{5}}|\log \beta| \beta^{\gamma},
$$

where $\gamma=(1+\alpha) /(1+\alpha / 2)$ and $|\theta| \leqslant 1$. To estimate the sum, we will use Lemma 
4. However, the bound for $S_{\mathrm{I}}$ is no longer valid, because $z-z_{j}$ may be arbitrarily small. Since $\left|z-\widetilde{z}_{j}\right|$ and $\left|z-z_{j}\right|$ are less than $\delta$ for all points $z_{j}$ in region I, we can use the bound (3.1) to estimate the first part of the sum in (4.2) and get

$$
\begin{aligned}
\left|S_{\mathrm{I}}\right| & \leqslant \sum_{z_{j} \in I_{1} \cup I_{2} \cup I_{3}}\left(\frac{C_{0}}{\delta}+\frac{C_{0}}{\delta}\right) \cdot M \cdot\left|B_{j}\right| \\
& \leqslant 32 \pi C_{0} C_{2}^{2} M \frac{\beta^{2}}{\delta} .
\end{aligned}
$$

By combining this result with Lemma 4, we conclude from Eq. (4.2) and Theorem 1 that

$$
\begin{aligned}
|\tilde{u}-u| & \leqslant 32 \pi C_{0} C_{2}^{2} M \beta^{\gamma}+C|\log \beta| \max _{j}\left|\tilde{z}_{j}-z_{j}\right|+C_{5}|\log \beta| \beta^{\gamma} \\
& \leqslant\left(32 \pi C_{0} C_{2}^{2} M+C C_{6}+C_{5}\right)|\log \beta| \beta^{\gamma-C t}
\end{aligned}
$$

for $\beta$ sufficiently small. This completes the first part of the proof. In the second part we let the distance from $z$ to supp $\xi$ be larger than $D$. We must, therefore, reconsider the derivation of Lemma 3. Since $\xi\left(z^{\prime}\right)$ vanishes for all $z^{\prime}$ in the disk $\left|z-z^{\prime}\right|<\delta$, we see that the third term in Eq. (3.2) is zero. Let $4 r_{1}<D$. To estimate the right-hand side of Eq. (3.3) we use Taylor's formula with remainder (as in $S_{\mathrm{II}}$ ) and get

$$
\begin{aligned}
|S| & \leqslant \sum_{j}\left[\frac{\sqrt{2} C_{0}}{\left(D-r_{1}\right)^{2}} \cdot r_{1} \cdot H r_{1}^{\alpha}\left|B_{j}\right|+\frac{1}{2} \frac{C_{0}}{\left(D-2 r_{1}\right)^{3}} \cdot 2 r_{1}^{\alpha} \cdot M \cdot\left|B_{j}\right|\right] \\
& \leqslant 6 \pi C_{0} C_{2}^{1+\alpha} H \beta^{1+\alpha}+18 \pi C_{0} C_{2}^{2} \frac{M}{D} \beta^{2} .
\end{aligned}
$$

The sum is taken over those $j$ for which $\int_{B_{j}}|\xi|$ is larger than zero. Thus the right-hand side of this inequality replaces the estimate of the truncation error in Lemma 3 . Since $\left|z-z_{j}+\theta\left(\tilde{z}_{j}-z_{j}\right)\right|$ is larger than $D-2 r_{1}$ for all $|\theta| \leqslant 1$, we infer from Eq. (4.2) by using the fundamental theorem of calculus and the bound (3.1) that

$$
\begin{aligned}
|u-\tilde{u}| & \leqslant \sum_{j} \frac{\sqrt{2} C_{0}}{\left(D-2 r_{1}\right)^{2}} \cdot\left|\tilde{z}_{j}-z_{j}\right| \cdot M \cdot\left|B_{j}\right|+|S| \\
& \leqslant\left[13 \pi C_{0} C_{6} M+6 \pi C_{0} C_{2}^{2} H+18 \pi C_{0} C_{2}^{2} \frac{M}{D}\right] \beta^{\gamma-C t} .
\end{aligned}
$$

We observe now that our last estimate is smaller than the right-hand side of the inequality (4.3) for $\beta$ sufficiently small, and this completes the proof.

Finally, we consider the convergence of the computed vorticity

$$
\widetilde{\xi}(z)=\sum_{j} \zeta_{\delta}\left(z-\widetilde{z_{j}}\right) \kappa_{j}
$$

to $\xi(z)$. In general it is not possible to show that $\widetilde{\xi}$ converges uniformly to $\xi$, because $\zeta_{\delta}$ may become unbounded at the origin, as in the case of the approximation (1.4).

However, even though the position $\widetilde{z}_{j}$ of the point vortices are computed by using the 
cutoff (1.4), we may interpolate the results by using a vorticity which corresponds to a different cutoff. In the proof below we choose the one corresponding to the approximation (1.5).

THEOREM 3. Let $\zeta_{\delta}(z)=1 / \pi \delta^{2}$ for $|z|<\delta$ and 0 otherwise, and let $\tilde{\xi}$ be given by Eq. (4.4). There exists a constant $C_{9}$ such that

$$
|\tilde{\xi}(z)-\xi(z)| \leqslant C_{9} \beta^{(\alpha / 2) /(1+\alpha / 2)}
$$

for $0 \leqslant t \leqslant T_{0}$ provided $\beta$ is sufficiently small and $\delta=\beta^{1 /(1+\alpha / 2)}$.

Remark. This result can be substantially improved, if it is assumed that $\xi$ and $\zeta_{\delta}$ are twice differentiable, but we shall not do so.

Proof. Let $z_{j}$ be the centroids of the $B_{j}$. We begin by decomposing $\xi(z)$ in a manner similar to Eq. (3.2)

$$
\begin{aligned}
\xi(z)= & \sum_{j} \zeta_{\delta}\left(z-z_{j}\right) \int_{B_{j}} \xi\left(z^{\prime}\right) d z^{\prime}+\sum_{j} \int_{B_{j}}\left[\zeta_{\delta}\left(z-z^{\prime}\right)-\zeta_{\delta}\left(z-z_{j}\right)\right] \xi\left(z^{\prime}\right) d z^{\prime} \\
& +\int_{\left|z-z^{\prime}\right|<\delta} \zeta_{\delta}\left(z-z^{\prime}\right)\left[\xi(z)-\xi\left(z^{\prime}\right)\right] d z^{\prime}
\end{aligned}
$$

Since $\xi$ is Hölder continuous, we can estimate the last term by $H \delta^{\alpha}$. To estimate the second term, which we denote by $S$, we cover the supp $\xi$ by a sequence of annuli, as in the proof of Lemma 3. If $z-z_{j}$ and $z-z^{\prime}$ are less than $\delta$ for all $z^{\prime}$ in $B_{j}$, then the integral over $B_{j}$ in the second sum of Eq. (4.5) is zero. The same conclusion holds if $z-z_{j}$ and $z-z^{\prime}$ are greater than $\delta$ for all $z^{\prime}$ in $B_{j}$. We can, therefore, estimate the sum $S$ by

$$
|S| \leqslant \sum_{k=n-1}^{n+1} \sum_{z \in I_{k}}\left[\frac{1}{\pi \delta^{2}}+\frac{1}{\pi \delta^{2}}\right] M \cdot\left|B_{j}\right| \leqslant 20 C_{2} M \frac{\beta}{\delta} .
$$

Let $C_{8}=H+20 C_{2} M$. Since $\delta^{\alpha}<\beta / \delta$, we conclude from Eq. (4.5) that $\xi(z)$ can be approximated uniformly and the error can be estimated by

$$
\left|\xi(z)-\sum_{j} \zeta_{\delta}\left(z-z_{j}\right) \kappa_{j}\right| \leqslant C_{8} \beta^{(\alpha / 2) /(1+\alpha / 2)} .
$$

Even if the vorticity $\xi$ is a smooth function, the error will be of order $\beta^{1 / 3}$, and the approximation is therefore quite crude. We can now compare $\tilde{\xi}$ with $\xi$ and have

$$
\tilde{\xi}-\xi=\sum_{j}\left[\zeta_{\delta}\left(z-\widetilde{z}_{j}\right)-\zeta_{\delta}\left(z-z_{j}\right)\right] \kappa_{j}+\theta C_{8} \beta^{(\alpha / 2) /(1+\alpha / 2)} .
$$

It follows from Theorem 1 that $\left|\widetilde{z}_{j}-z_{j}\right| \leqslant r_{1}$ for all $z_{j}$. To complete the proof we estimate the sum in (4.6) by using the same argument as for $S$ and we take $C_{9}=2 C_{8}$.

5. Numerical Experiments. To support the theory presented in the previous sections we have carried out a large number of numerical experiments. The preliminary calculations were done on the CDC-6400 at the University of California, Berkeley, while the results presented here were obtained on the CDC-7600 at Lawrence Berkeley Laboratory. As initial vorticity we choose $\xi(z)=1-|z|$ for $|z| \leqslant 1$ and zero otherwise. 
The solution of Euler's equations can be found explicitly and satisfies the smoothness assumptions used in this paper. To discretize the problem we covered $|z| \leqslant 1$ by a uniform mesh with meshlength $\beta$. In the experiments presented below $\beta$ lies in the interval $[0.1,0.5]$. The mesh was placed such that $z=0$ became the center of gravity (the centroid) of one of the small squares. To find the strength $\kappa_{j}$ of the point vortex in the square $B_{j}$ we calculated the double integral $\int_{B_{j}} \xi d x d y$ numerically by using the trapezoidal rule with meshlength $\beta / 10$. To reduce the costs of the calculations we have excluded those vortices for which $\kappa_{j}<1 / 30 \beta^{3}$. The bulk of our experiments were done with the cutoff (1.4). Finally, the ordinary differential equations (1.8), (1.9) were solved by using the classical fourth order Runge-Kutta method.

We have used several ways of measuring the error. Since the solution of the Euler's equations is stationary and rotational invariant, we can compare the positions of the point vortices at time $t$ with the positions of the material points which follow the streamlines. Thus we compute

$$
\beta \sqrt{\sum_{j}\left(\text { computed } z_{j}-\operatorname{exact} z_{j}\right)^{2}} .
$$

The sum is taken over all $j$ for which $\kappa_{j} \geqslant \beta^{3} / 30$, and it is an estimate of the error in $L_{2}$ over the support of $\xi$. Another way of estimating the error is to compare the position of the point vortices with the centers of gravity (the centroids) of the blobs $B_{j}$ at time $t$. Although the stream-function $\Phi_{t}$ can be given explicitly, we have not been able to find a convenient expression in closed form for the center of gravity. Consequently we have estimated $\beta^{-2} \int_{B(t)} z d x d y$ by evaluating the double integral $\beta^{-2} \int_{B(0)} \Phi_{t}\left(z^{\prime}\right) d z^{\prime}$. Note that $B(0)$ is a square. This calculation was done by using the rectangle rule with meshlength $\beta / 20$. Thus, we have also computed

$$
\beta \sqrt{\sum_{j}\left(\text { computed } z_{j}-\text { centroid } B_{j}\right)^{2}} \text {. }
$$

According to Lemma 2, there should only be a small difference between the expressions (5.1) and (5.2); but we have found that the two error estimates behave quite differently. Finally, we have studied the pointwise convergence of the vortex method.

It follows from the proof of Theorem 1 that with the above choices of initial vorticity and of the cutoff, the vortex method will converge for $0 \leqslant t \leqslant 0.00062$ provided $\beta$ is sufficiently small. This is less than impressive, and we have investigated the algorithm for $0 \leqslant t \leqslant 6$. In this time the vortices at $|z|=1$ travel one radian, while those near $z=0$ travel three radians. We begin with the cutoff $\delta=\beta^{2 / 3}$ since our theory gives preference to this choice. It follows from Table 1 that the error in the vortex method increases linearly in time. There is no hint of any exponential loss of accuracy as $t$ increases.

The time-step in the Runge-Kutta method is $\Delta t=1$. By comparing the numerical solution of (1.8), (1.9) with $\Delta t=1$ with the solution for $\Delta t=1 / 2$ and $1 / 4$, we estimate that the error due to the numerical solution of the differential equations is less than one percent of the error due to the discretization. Finally, Table 1 reveals that the difference between the positions of the point vortices and the centroids is 
TABLE 1. Linear growth of the error

$$
\text { Cutoff (1.4), } \delta=\beta^{2 / 3}, \beta=1 / 10, \Delta t=1
$$

\begin{tabular}{l|lll}
\hline & $t=2$ & $t=4$ & $t=6$ \\
\cline { 2 - 4 } (5.1): Comp. - exact & 0.0163 & 0.0328 & 0.0494 \\
(5.2): Comp. - centroid & 0.0147 & 0.0296 & 0.0446 \\
\hline
\end{tabular}

\begin{tabular}{|c|c|c|c|c|}
\hline TABLE & \multicolumn{3}{|c|}{ Cutoff (1.4), $\delta=\beta^{2 / 3}, t=1, t=6$} & \\
\hline & $\beta=1 / 2$ & $\beta=1 / 3$ & $\beta=1 / 4$ & $\beta=1 / 5$ \\
\hline Comp. - exact & 1.23 & 1.32 & 1.39 & 1.40 \\
\hline Comp. - centroid & 1.06 & 1.21 & 1.30 & 1.33 \\
\hline
\end{tabular}

smaller than the difference between the point vortices and the material points on the streamlines. We have found that in general this holds even pointwise, but it may fail if the calculations are extended over long time intervals. The reason is that the blobs get quite distorted and the center of gravity for a blob may lie far from the blob. For a fixed time this last phenomenon disappears as $\beta$ tends to zero.

To estimate the rate of convergence for the vortex method, we have used Richardson's extrapolation with the meshlengths $\beta$ and $\beta / 2$. If we ignore the exponential loss of accuracy, which we believe is a technical artifact, then we find from Theorem 1 that the rate of convergence should be 1.33 . This is borne out in the experiments presented in Table 2 .

In all our experiments with the cutoff (1.4), we have found that the rate of convergence measured in the norm (5.1) is larger than the rate of convergence measured in the norm (5.2), even though the values of (5.2) are consistently smaller than the values of (5.1). We have not been able to explain this phenomenon. The estimate in Table 2 of the rate of convergence is calculated at $t=6$, but we have observed that the rate of convergence is decaying by roughly 0.01 per unit time interval. This also remains inexplicable. It seems to be independent of the smoothness of the initial vorticity, of the meshlength $\beta$, and of the timestep $\Delta t$. On the other hand, for some cutoffs, see e.g., (5.7), the rate of convergence may increase as time progresses.

One may argue that the superlinear convergence of the vortex method is due to a lucky cancellation caused by the rotational symmetry of the initial vorticity. To counter this objection we have replaced the cone $\xi=1-|z|$ by a pyramid with center 
at $z=0$ and height one. The vertices of the pyramid are placed at $x= \pm 1$ and $y= \pm 1$. The solution of the Euler equations with this initial vorticity seems to be unknown. To estimate the rate of convergence we have used Richardson's extrapolation with three meshlengths $\beta, 2 / 3 \beta$ and $1 / 2 \beta$. If the computed solution, i.e. $z_{j}$, is equal to the exact solution plus $\beta^{4 / 3} e(x, y, t)$ plus higher order terms, then

$$
\frac{\| \text { comp: } \beta-\text { comp: } \frac{2}{3} \beta \|}{\| \text { comp: } \frac{2}{3} \beta-\text { comp: } \frac{1}{2} \beta \|} \sim \frac{\beta^{4 / 3}-\left(\frac{2}{3} \beta\right)^{4 / 3}}{\left(\frac{2}{3} \beta\right)^{4 / 3}-\left(\frac{1}{2} \beta\right)^{4 / 3}} \doteq 2.25 \text {. }
$$

We choose the norm in (5.3) to be the discrete $l_{2}$ norm of the differences between the vortices which have the same initial positions. With $\beta=1 / 4$ and $t=0$, the three meshes overlap at all points $(x, y)$ for which $x, y=0, \pm 1 / 2, \pm 1$. In our numerical experiment we found that the first quotient in (5.3) is equal to 2.26, 2.17 and 2.22 for $t=2,4$, and 6 , respectively. These results indicate that the rate of convergence, which we have observed with the initial vorticity $\xi=1-|z|$, may be trusted.

We will now present some calculations with initial vorticity $\xi=1-|z|$, which indicate that the rate of convergence of the vortex method may be of the second order. This has come as a great surprise to us. In the proof of Lemma 3, the optimal choice of $\delta$ was determined by letting $\delta^{2} \sim \beta^{2} / \delta$. Here we assume that the solution $\xi$ is at least Lipschitz continuous. Thus, if $\delta=\beta^{p}$, then we expect that the rate of convergence should be $2 p$ for $1 / 2<p \leqslant 2 / 3$ and $2-p$ for $2 / 3 \leqslant p<1$. However, there is no indication in Figure 1 of the last branch. This leads us to conjecture that the factor $\beta^{2} / \delta$ in our estimates is due to an imperfect technique, and should really be replaced by $\beta^{2}$.

It follows from Table 3 that the numerical error for the vortex method is smallest for $p=1$. Thus, in practice there is no reason to take $\delta=\beta^{p}$ with $p<1$. However, if $\delta=\beta$, then our technique cannot be used to prove the convergence of the vortex method even for a short time interval.

TABLE 3. Errors in the vortex method

$$
\text { Cutoff (1.4), } \delta=\beta^{p}, \Delta t=1, t=6
$$

\begin{tabular}{l|cccccc}
\hline & $p=1 / 6$ & $1 / 3$ & $1 / 2$ & $2 / 3$ & $5 / 6$ & 1 \\
\cline { 2 - 7 } Comp. - exact, $\beta=1 / 4$ & 0.486 & 0.347 & 0.242 & 0.176 & 0.126 & 0.0946 \\
Comp. - exact, $\beta=1 / 8$ & 0.391 & 0.220 & 0.121 & 0.0673 & 0.0396 & 0.0256 \\
\hline
\end{tabular}

We will now give a heuristic explanation of the results in Figure 1 and Table 3. Assume that our conjecture is valid. Then the last term in Eq. (3.2) is the dominating term in the truncation error. It is easy to show that if the solution $\xi$ is three times differentiable, then the last term in Eq. (3.2) is equal to

$$
C \delta^{2}\left(\begin{array}{c}
\xi_{y} \\
-\xi_{x}
\end{array}\right)+O\left(\delta^{4}\right)
$$




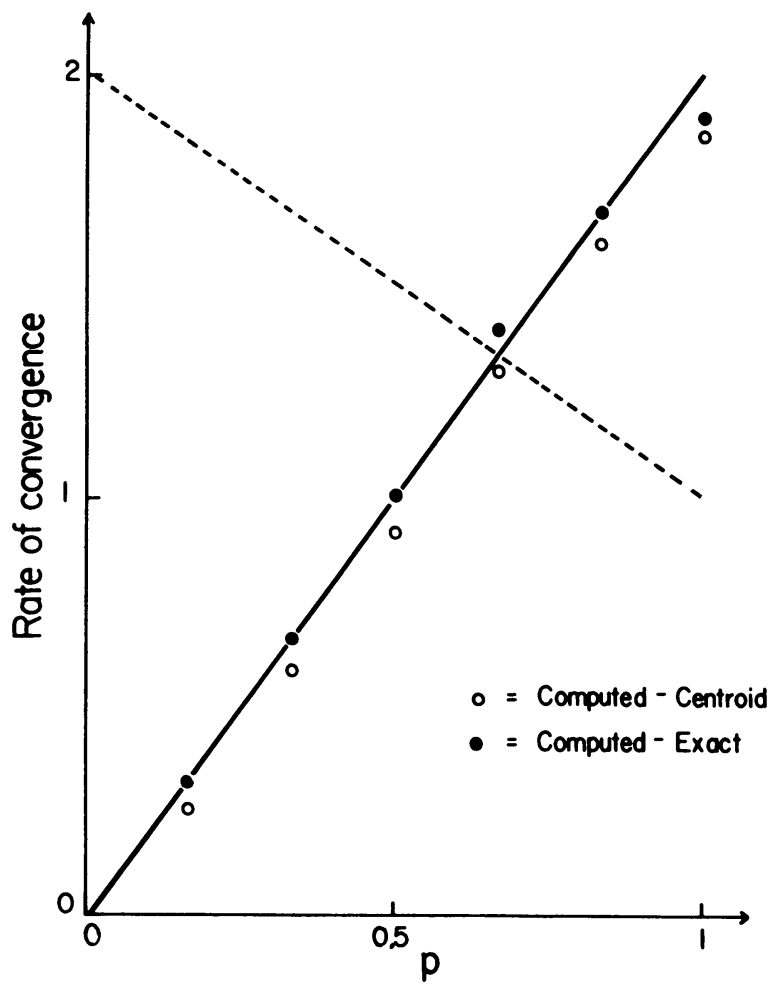

FIGURE 1. Rate of convergence for $0<p \leqslant 1$ with $\delta=\beta^{p}$

Cutoff (1.4), $\beta=1 / 4$ and $1 / 8, \Delta t=1$ and $t=6$

where the constant $C$ depends on the particular cutoff. Thus, if the vortex method is actually stable, and not weakly unstable as in Lemma 4, then the error should be expected to be of order $\beta^{2 p}$. This explains the results in Figure 1. Note, however, that the solution $\xi$ is not three times differentiable at $z=0$ and $|z|=1$. The decay of the errors in Table 3 can be well described by const $\cdot \beta^{a p}$, where $a=$ 1.43 for $\beta=1 / 4$ and $a=1.60$ for $\beta=1 / 8$. Although neither of these results are consistent with (5.3), they do explain why the observed rate of convergence in Figure 1 is very close to $2 p$. The constant $C$ in the expression (5.4) is equal to $1 / 12$, $1 / 8$, and $3 / 40$ for the cutoffs (1.4) to (1.6), respectively. Thus we expect that the cutoff (1.4) which has been used by Chorin [2] and Shestakov [11] should be superior to the cutoff (1.5), which has been used by Milinazzo and Saffman [8], while the cutoff (1.6) should be the best of the three. This is clearly borne out in Table 4 .

TABLE 4. Errors for different cutoffs

Initial vorticity $\xi=1-|z|, \delta=\beta^{2 / 3}, \Delta t=1, t=6$

\begin{tabular}{r|lll}
\hline Cutoff: & $(1.4)$ & $(1.5)$ & $(1.6)$ \\
\cline { 2 - 4 } Comp. - exact, $\beta=1 / 4$ & 0.176 & 0.237 & 0.153 \\
Comp. - exact, $\beta=1 / 8$ & 0.0673 & 0.0936 & 0.0609 \\
\hline
\end{tabular}


The quotients between the errors are very close to those predicted by the expression (5.3). Finally, we mention that for the next cutoff in the sequence, the constant $C$ is equal to $1 / 20$; and this cutoff must, therefore, be expected to be even better.

It is natural to ask whether it is possible to construct a cutoff such that the constant $C$ in (5.4) is zero. It is easy to show that if the cutoff corresponds to a blob of vorticity which is everywhere nonnegative, then $C$ must be positive. However, the constant $C$ vanishes for the following two cutoffs:

(5.7) $G_{\delta}=\frac{1}{2 \pi}\left[5\left(1-\frac{r^{2}}{\delta^{2}}\right)-\frac{45}{4}\left(1-\frac{r^{4}}{\delta^{4}}\right)+\frac{56}{5}\left(1-\frac{r^{5}}{\delta^{5}}\right)-\frac{10}{3}\left(1-\frac{r^{6}}{\delta^{6}}\right)-\log \delta\right]$.

We note that the kernels $K_{\delta}$, which correspond to the two cutoffs, satisfy the inequality (3.1). The performance of (5.6) and (5.7) are comparable and the errors for the two cutoffs are in general considerably smaller than the errors for the cutoffs (1.4), (1.6). The new cutoffs also have theoretical advantages. If the solution $\xi$ is three times differentiable, then we conclude from (5.4) that the optimal choice of $\delta$ is obtained by letting $\delta^{4}$ be of the same order as $\beta^{2} / \delta$. Thus, if $\delta=\beta^{0.4}$, then the expected rate of convergence should be $\beta^{1.6}$. We have tested the cutoff (5.7) and report faithfully the results in Table 5.

TABLE 5. Rate of convergence

Cutoff (1.5), $\delta=\beta^{p}, \beta=1 / 4$ and $1 / 8, \Delta t=1, t=6$

\begin{tabular}{l|cccccc}
\hline & $p=1 / 6$ & $1 / 3$ & $1 / 2$ & $2 / 3$ & $5 / 6$ & 1 \\
\cline { 2 - 7 } Comp. - exact & 0.79 & 1.52 & 2.05 & 2.53 & 1.81 & 1.59 \\
Comp. - centroid & 0.48 & 1.29 & 2.02 & 2.59 & 1.62 & 1.38 \\
\hline
\end{tabular}

The initial vorticity was chosen as a rotated cubic spline with center at $z=0$, height one, and with knots at $|z|=1 / 2$ and $|z|=1$. To reduce the cost of the calculation we have excluded those vortices for which $\kappa_{j}<1 / 10 \beta^{5}$. For $\delta=\beta^{p}$ and $p \leqslant 1 / 2$ the rate of convergence seems to be $\beta^{4 p}$ while the rate of convergence for $1 / 2<$ $p<1$ remains mysterious. We would have expected second order accuracy in this interval. Finally, we have observed that for $1 / 2 \leqslant p \leqslant 1$ the rate of convergence may change by as much as 0.1 per unit time interval.

Acknowledgments. The authors thank Alexandre J. Chorin for helpful discussions. The work of the first author was carried out in part at the Lawrence Berkeley Laboratory under the auspices of the U. S. Energy Research and Development 
Administration. The paper was written while the second author was visiting the University of California, Berkeley, with a fellowship from the Consiglio Nazionale delle Ricerche, Italy.

Department of Mathematics

Lawrence Berkeley Laboratory

University of California

Berkeley, California 94720

Istituto di Matematica

Universita di Genova

16132 Genova, Italy

1. A. K. BATCHELOR, Introduction to Fluid Dynamics, Cambridge Univ. Press, London, 1967.

2. A. J. CHORIN, "Numerical study of slightly viscous flow," J. Fluid Mech., v. 57, 1973, pp. 785-796.

3. A. J. CHORIN \& P. S. BERNARD, "Discretization of a vortex sheet, with an example of roll-up," J. Computational Phys., v. 13, 1973, pp. 423-429.

4. T. E. DUSHANE, "Convergence for a vortex method for solving Euler's equation,"

Math. Comp., v. 27, 1973, pp. 719-728.

5. P. HARTMAN, Ordinary Differential Equations, Wiley, New York, 1964.

6. T. KATO, "On classical solutions of the two-dimensional non-stationary Euler equation," Arch. Rational Mech. Anal., v. 25, 1967, pp. 188-200.

7. F. J. McGRATH, "Nonstationary plane flow of viscous and ideal fluids," Arch. Rational Mech. Anal., v. 27, 1968, pp. 329-348.

8. F. MILINAZZO \& P. G. SAFFMAN, "The calculation of large Reynolds number twodimensional flow using discrete vortices with random walk," J. Computational Phys., v. 23, 1977, pp. 380-392.

9. D. W. MOORE, The Discrete Vortex Approximation of a Finite Vortex Sheet, California Inst. of Tech. Report AFOSR-1 804-69, 1971.

10. L. ROSENHEAD, "The formation of vortices from a surface of discontinuity," Proc. Roy. Soc. London Ser. A, v. 134, 1932, pp. 170-192.

11. A. I. SHESTAKOV, Numerical Solution of the Navier-Stokes Equations at High Reynolds Numbers, Ph. D. Thesis, Univ. of California, Berkeley, Calif., 1975.

12. H. TAKAMI, Numerical Experiment with Discrete Vortex Approximation, with Reference to the Rolling Up of a Vortex Sheet, Dept. of Aero. and Astr., Stanford University Report SUDAER-202, 1964.

13. F. L. WESTWATER, Aero.Res. Coun., Rep. and Mem. \# 1692, 1936. See also Batchelor [1, p. 590].

14. W. WOLIBNER, "Un théorème sur l'existence du mouvement plan d'un fluide parfait, homogène, incompressible, pendent un temps infiniment long," Math. Z., v. 37, 1933, pp. 698726. 